\title{
Diagnostic yield is dependent on monitoring duration. Insights from a full-disclosure mobile cardiac telemetry system
}

\author{
Marek J Dziubiński ${ }^{1}$, Natan E Napiórkowski', Olga Witkowska', Michał A Świecak', Agnieszka M Grotek', \\ Linda SB Johnson ${ }^{2,3}$
}

${ }^{1}$ Medicalgorithmics SA, Warszawa, Poland

2 Department of Clinical Sciences, Lund University, Malmö, Sweden

${ }^{3}$ Department of Imaging and Functional Studies, Skåne University Hospital, Malmö, Sweden

Correspondence to:

Marek J Dziubiński,

Medicalgorithmics $S A$,

Al. Jerozolimskie 81, 02-001

Warszawa, Poland,

phone: +48228251249,

e-mail:m.dziubinski@

medicalgorithmics.com

Copyright by the Author(s), 2022

DOI: 10.33963/KP.a2021.0182

\section{Received:}

September 27, 2021

Accepted:

December 15, 2021

Early publication date:

December 16, 2021

\section{A B S T R A C T}

Background: Despite the advancement of electrocardiogram (ECG) monitoring methods, the most important factor influencing diagnostic yield (DY) may still be monitoring duration. Ambulatory ECG monitoring, typically with $24-48$ hours duration, is widely used but may result in underdiagnosis of rare arrhythmias.

Aims: This study aimed to examine the relationship between the DY and monitoring duration in a large patient cohort and investigate sex and age differences in the presentation of arrhythmias.

Methods: The study population consisted of 25151 patients ( $57.8 \%$ women; median [interquartile range, IQR], 71 [64-78] years), who were examined with mobile cardiac telemetry during 2017 in the United States, using the PocketECG ${ }^{\mathrm{TM}}$ that continuously transmits a signal on a beat-to-beat basis. We investigated the occurrence of atrial fibrillation at a burden of both $\leq 1 \%$ (atrial fibrillation $[\mathrm{AF}], \leq 1 \%$ ) and $\leq 10 \%$ (AF $\leq 10 \%)$, premature ventricular contractions (PVC; $>10000$ per 24 hours), non-sustained ventricular tachycardias (nsVT), sustained ventricular tachycardias (VT $\geq 30$ seconds), atrioventricular blocks (AVB), pauses of $>3$ seconds duration, and bradycardia (heart rate $<40$ beats per minute for $\geq 60$ seconds).

Results: The median (IQR) recording duration was 15.4, 8.2-28.2) days. The DY increased gradually with monitoring duration for all types of investigated arrhythmias. Compared to DY after up to 30 days of monitoring, a standard 24 hours monitoring resulted in DY for males/females of $20 \% / 18 \%$ for AF $\leq 1 \%, 29 \% / 28 \%$ for $\mathrm{AF} \leq 10 \%, 45 \% / 40 \%$ for PVCs, $17 \% / 11 \%$ for $\mathrm{nsVT}, 17 \% / 11 \%$ for VT $\geq 30$ seconds, 49\%/42 for AVB, 27\%/20\% for pauses, 36\%/29\% for bradycardia.

Conclusion: A substantial number of patients suffering from arrhythmias may remain undiagnosed due to insufficient ECG monitoring time.

Key words: ECG monitoring, diagnostic yield, mobile cardiac telemetry monitoring, arrhythmias.

\section{INTRODUCTION}

Despite significant advances in electrocardiogram (ECG) monitoring methods, one of the most important factors influencing arrhythmia diagnostic yield is still the duration of the monitoring, which varies widely from as short as 10 seconds (wearable consumer devices) to several months or years (implanted devices). Analysis of ECG data may be immediate or postponed. Uncertainty remains regarding necessary monitoring durations in specific clinical situations, such as for detection of infrequently occurring atrial fibrillation, cardiogenic syncope, or suspicion of ventricular arrhythmias, although it has been suggested that longer monitoring durations are necessary [1,2]. Short recording durations imply a risk of missing infrequent arrhythmic episodes [3]. Mobile Cardiac Telemetry (MCT) addresses these drawbacks, 
WHAT'S NEW?

The manuscript draws on a very large database of up to 30-day-long full disclosure electrocardiogram (ECG) recordings with beatto-beat analysis to show how the diagnostic yield for several clinically important arrhythmias depends on monitoring duration. The detailed analysis of the ECG allows us to present data on diagnostic yield for arrhythmias which have been understudied.

by providing monitoring for an extended period (several weeks) with online access to the diagnostic findings, as well as providing a detailed ECG analysis that enables the assessment of other clinically relevant measures of heart rate and rhythm [4].

The primary aim of the present study was to use MCT recordings of up to 30 days to determine the impact of the recording duration on the diagnostic yield for several clinically important arrhythmias: atrial fibrillation (AF) with burdens below $\leq 1 \%$ and $\leq 10 \%$, frequent premature ventricular contractions (PVCs), non-sustained (nsVT) and sustained ventricular tachycardias (VT $\geq 30$ seconds), $2^{\text {nd }}$ and $3^{\text {rd }}$-degree atrioventricular blocks (AVB), pauses, and bradycardia [5]. The secondary objective was to investigate sex-specific and age-related differences in the presentation of all arrhythmias.

\section{METHODS}

The study consists of 25151 up to 30 days long ECG recordings performed with the PocketECG ${ }^{\text {TM }}$ device (MediLynx Cardiac Monitoring, Plano, TX, USA) in clinical practice in the United States in 2017. All patients included in the study were 18-100 years old, and analyses were performed according to pre-specified age strata with the cut-off point at the standard age of retirement in the United States set at 65 years. Besides age, sex, and indication for monitoring, no details concerning clinical characteristics and the patient's medical history were available due to the nature of the database. The most frequent ICD-10 codes given as indication for monitoring were R00.2 Palpitations ( $n=12$ 673), 149.8 Other cardiac arrhythmias $(n=4701)$, R55 Syncope and Collapse ( $n=4428)$, 148.0 Paroxysmal atrial fibrillation ( $n=3479$ ), R42 Dizziness and Giddiness ( $n=3237), 148.91$ Unspecified atrial fibrillation $(n=2372)$, R00.1 Bradycardia ( $n=1872)$, R06.00 Dyspnea ( $n=1716)$, R00.00 Tachycardia ( $n=1261)$, G45.9 Transient ischemic attack ( $\mathrm{n}=1247$ ), 147.1 Supraventricular tachycardia $(n=930), 148.92$ Unspecified atrial flutter $(n=748)$, 145.89 Other conduction disorders ( $n=669)$, 148.1 Persistent atrial fibrillation $(n=662), 145.9$ Conduction disorder ( $n=481)$. All patient information was de-identified, the research reported in this paper adhered to ISO/IEC 27001:2013 "Information technology - Security techniques - Information security management systems - Requirements" and the Ethics Review Board of Sweden has waived the need for ethics approval for studies using this data (decision no. 2019-03227).
PocketECG ${ }^{\mathrm{TM}}$ is a Food and Drug Administration (FDA) approved mobile ECG recorder that continuously transmits a full ECG signal, with real-time algorithmic detection of arrhythmias and validation by trained ECG technicians [6, 7]. Data were analyzed in a beat-by-beat manner, which enabled precise assessment of the number of arrhythmic episodes together with the exact time of occurrence and duration of each episode. Pocket ECG is most often prescribed for 30 days. The real-time analyses of the ECG signal are available to the referring physician throughout the ECG monitoring, and this allows for the clinicians to shorten or extend the monitoring duration based on the ongoing results. Therefore, the included recording durations vary within the investigated sample.

\section{Endpoint ascertainment}

The diagnostic yield that was measured at different monitoring durations included arrhythmias. All arrhythmias were algorithmically detected and manually verified. AF was defined as $\geq 30$ seconds of irregular ventricular activity without $P$ waves. We examined all patients with paroxysmal and persistent AF and selected for analysis only patients with a low AF burden. For these analyses, patients with a total $A F$ burden $\leq 1 \%$ and $\leq 10 \%$ were included, to identify a subset with infrequent arrhythmia for whom necessary monitoring duration is not known. PVCs were defined as wide-QRS premature beats (duration greater than $120 \mathrm{~ms}$ ) that were not preceded by a P wave and were followed by a complete pause before the next regular heartbeat [8]. Frequent PVCs were defined as $\geq 10000$ PVCs/24 hours [9]. Sustained ventricular tachycardia was defined as a ventricular tachycardia $>100$ bpm lasting at least 30 seconds [10]. Non-sustained (nsVT) was defined as 4 or more consecutive beats arising below the atrioventricular node with an RR interval of $<600 \mathrm{~ms}$ ( $>100 \mathrm{bpm}$ ) and lasting $<30$ seconds [11]. PocketECG system distinguishes triplets from VT, and this distinction was maintained in analyses. Second-degree AVB was defined as delay or disturbance in the transmission of an impulse and sub-classified into Mobitz type 1 when there was a progressive prolongation of the PR interval culminating in a non-conducted $\mathrm{P}$ wave, and Mobitz type 2 when there were intermittent non-conducted $P$ waves without a pattern [12]. Third-degree AVB was defined as an absence of all AV nodal conduction [12]. A pause was defined as an RR-interval longer than 3 seconds, and bradycardia was defined as a ventricular rate $<40 \mathrm{bpm}$ lasting for at least 60 seconds [13]. 


\section{Statistical analysis}

All analyses were performed in the Python3 language with Anaconda environment for Windows, version 3.8, and using the Pandas, NumPy, and SciPy packages. Kaplan-Meier estimates with $95 \%$ confidence intervals $(\mathrm{Cl})$ were calculated using the Lifelines Python package [14].

The normality of continuous variables was assessed visually, and normally distributed continuous variables were reported as means (standard deviation [SD]) while skewed variables were reported as medians (interquartile range [IQR]). Dichotomous variables (sex and arrhythmia occurrence) were reported as counts (percentages). Arrhythmia occurrence of each specific arrhythmia event of interest was also handled as time-to-event data, with the time to an event defined as days between the initiation of recording until the first occurrence of each arrhythmia of interest. The cumulative DY, according to monitoring duration, was calculated for each consecutive day of monitoring using
Kaplan-Meier curves with recording duration as the time scale in which the arrhythmia of interest was considered the outcome event. Subjects were censored at the time of the end of monitoring, either after 30 days of monitoring or at the time registration ended if this occurred before 30 days of monitoring had ceased [15]. Ninety-five percent $\mathrm{Cl}$ were calculated using standard errors derived using Greenwood's Exponential formula [16]. Comparisons of diagnostic yield between men and women were performed using the log-rank test [17]. Diagnostic yield was calculated as a percentage of diagnosed patients after each day of monitoring relative to the total number of diagnosed patients after 30 days of monitoring, with reporting of diagnostic yield values after 24 hours, 48 hours, 5 days, 7 days, and 14 days compared to the diagnostic yield after 30 days. Comparisons of the number of patients with investigated arrhythmia was performed using the $X^{2}$ test (Figures 1 and 2 ). $P$-values $<0.05$ were considered statistically significant.
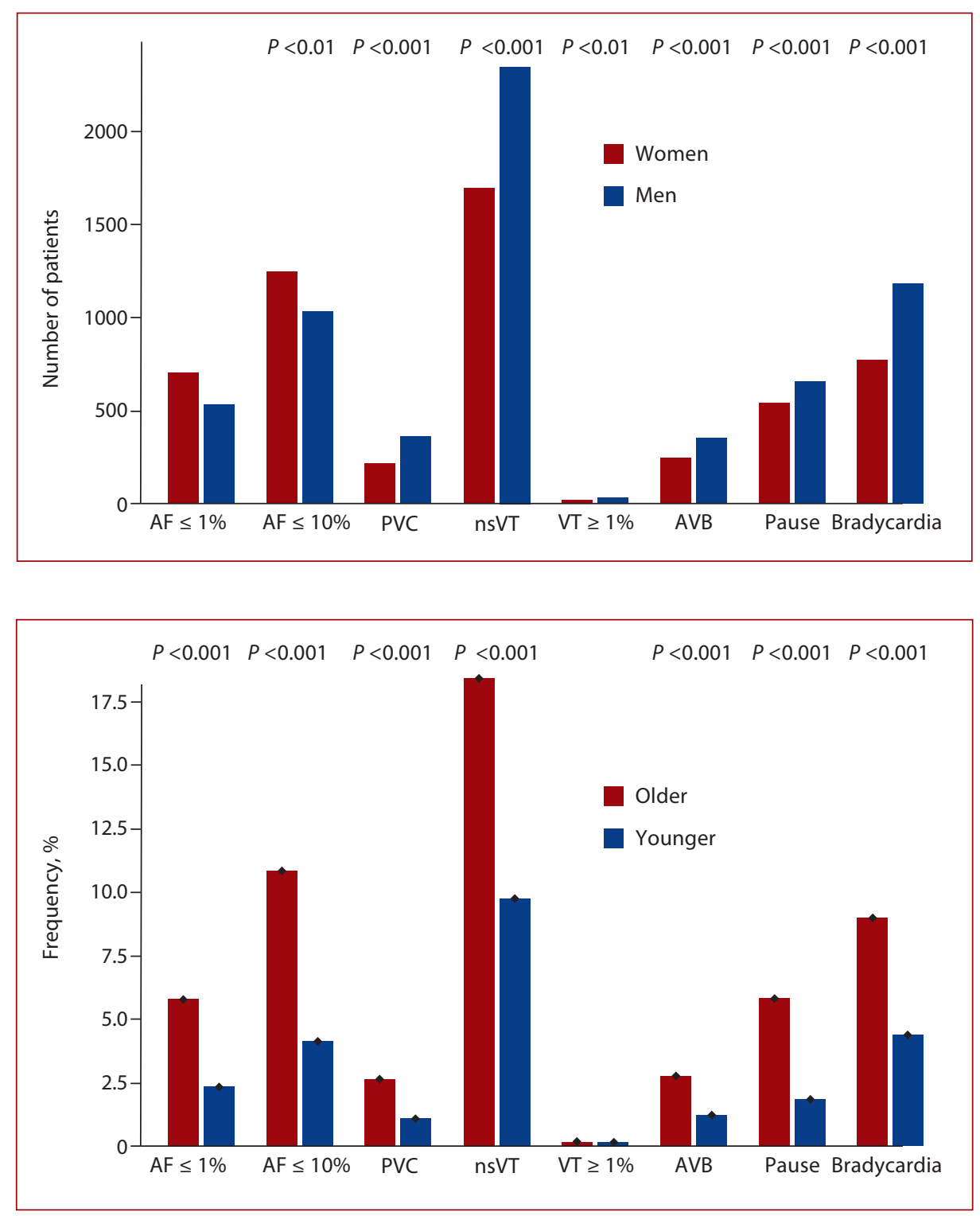

Figure 1. Frequencies of occurrences of different arrhythmias among women and men. Number of patients diagnosed with atrial fibrillation with burden $\leq 1 \%$ and $\leq 10 \%$, premature ventricular contractions, non-sustained ventricular tachycardias, ventricular tachycardias, atrioventricular blocks, pauses, and bradycardia Abbreviations: see Table 1
Figure 2. Frequencies of occurrences of different arrhythmias among younger (18-64 years) and older patients ( $\geq 65$ years). Number of patients diagnosed with atrial fibrillation with burden $\leq 1 \%$ and $\leq 10 \%$, premature ventricular contractions, non-sustained ventricular tachycardias, ventricular tachycardias, atrioventricular blocks, pauses, and bradycardia

Abbreviations: see Table 1 


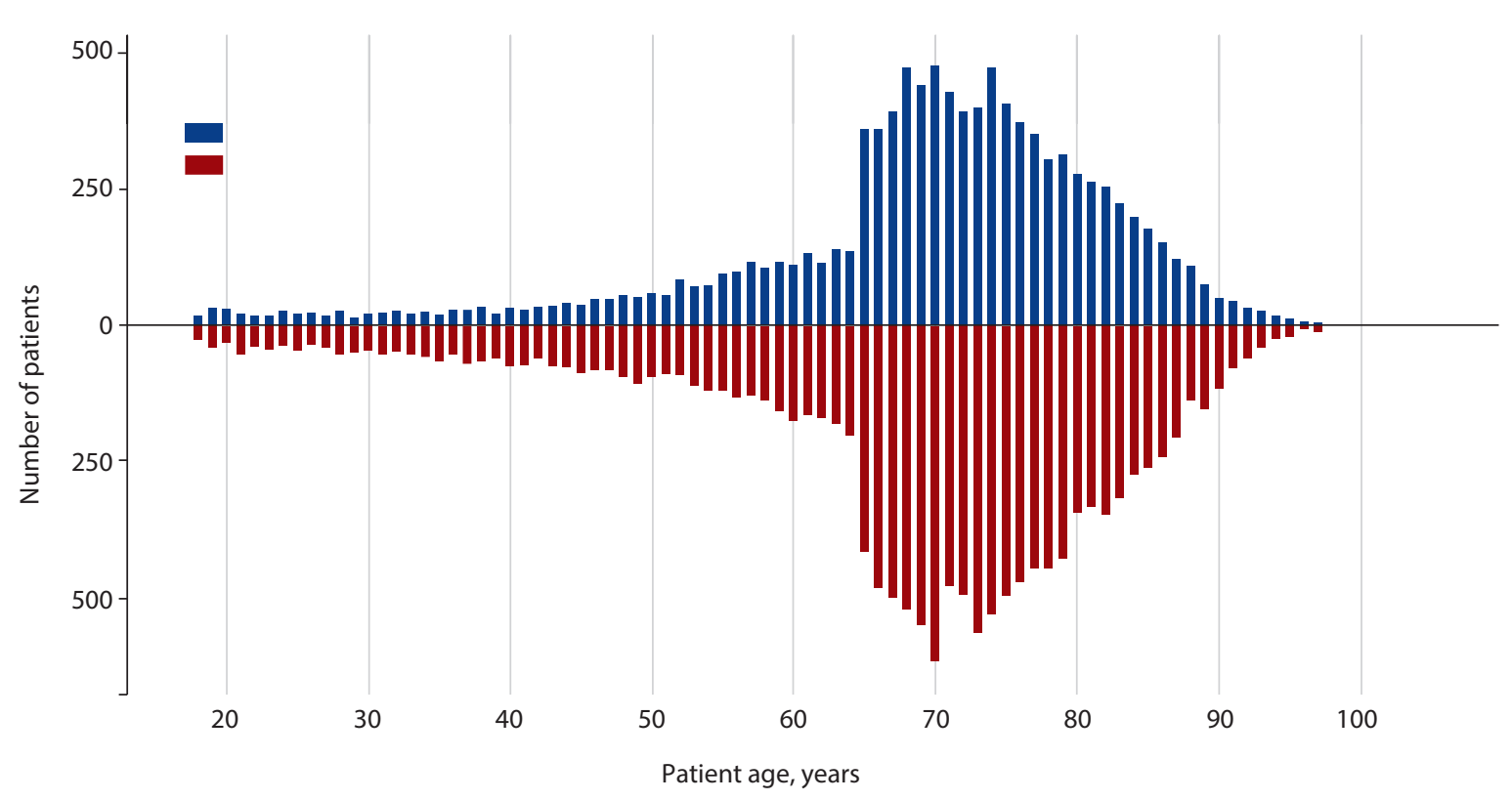

Figure 3. Age distribution among women (red) and men (blue) within the investigated sample

\section{RESULTS}

The study population consisted of 14543 (57.8\%) women and 10608 (42.2\%) men. Figure 3 presents the distribution of age in the investigated sample. The median (IQR) age was 71 (64-78) years, and the median recording duration (IQR) was 15.4 (8.2-28.2) days. The mean (SD) active monitoring duration per recording day was 20.27 (3.57) hours.

At least one arrhythmia of interest was found in 10799 patients (42.9\%), of whom $44.2 \%$ were women ( $n=4775$ ). All investigated arrhythmias were significantly more frequent in men than in women (all $P<0.01$, Figure 1) except for AF. The probability of a diagnosis of any arrhythmia of interest was higher for patients $\geq 65$ years compared to younger patients (all $P<0.001$, Figure 2 ).

\section{Monitoring duration affects diagnostic yield}

Tables 1 and 2 report diagnostic yield after 24 hours, 48 hours, 5 days, 7 days, and 14 days of monitoring by sex. Longer ECG monitoring resulted in higher cumulative diagnostic yield of all arrhythmias, but the strength of the association between diagnostic yield and monitoring time varied. A single day of monitoring diagnosed almost half of the patients with AVB, but fewer than 1 in 5 patients with AF. Two days of monitoring detected approximately half of patients with frequent PVCs, but fewer than a third of AF patients with $\leq 1 \%$ burden.

\section{Sex differences in diagnostic yield}

Sex differences in overall diagnostic yield, after the full monitoring duration, are presented in Figure 4. We observed that men, as compared to women, had significantly higher cumulative DY of frequent PVCs (the log-rank test, 4.135; $P=0.042$ ), AVB (the log-rank test, 5.731; $P=0.017$ ), and bradycardia (the log-rank test, 11.920; $P=0.001$ ). For all other arrhythmias, the cumulative diagnostic yield was comparable in men and women.

\section{Age differences in diagnostic yield}

The association between age and cumulative diagnostic yield of different arrhythmias is presented in Figure 5. Older subjects ( $\geq 65$ years) had a significantly higher cumulative diagnostic yield of AVB (the log-rank test, 11.981; $P=0.001$ ), and younger subjects (18-64 years) had a significantly higher cumulative diagnostic yield of sustained ventricular tachycardias (the log-rank test, 9.323; $P=0.002$ ). Otherwise, the cumulative diagnostic yield was comparable in older and younger subjects.

\section{CONCLUSION}

The cumulative diagnostic yield increased with ECG monitoring duration regardless of the type of the investigated arrhythmia. The diagnostic yield after 48 hours of monitoring, the typical duration of traditional Holter monitoring, rarely exceeded $50 \%$ of the diagnostic yield after 30 days of monitoring, implying that at least half of the patients might remain undiagnosed with a traditional Holter monitoring despite suffering from cardiac arrhythmias that could be diagnosed with longer monitoring durations. Furthermore, even after 14 days of monitoring $10 \%$ of patients had not been diagnosed.

We found substantial differences in the frequencies of arrhythmias between women and men. Although more women were examined with ECG monitoring, more men were diagnosed with arrhythmias, which is in accordance with previous research [18]. The finding that the diagnostic yield of AVB, frequent PVCs, and bradycardia was signifi- 
Table 1. Diagnostic yield in women calculated after 24 hours, 48 hours, 5, 7, and 14 days

\begin{tabular}{|c|c|c|c|c|c|c|c|c|c|}
\hline \multicolumn{2}{|c|}{ Arrhythmia } & AF $\leq 1 \%$ & $A F \leq 10 \%$ & PVCs & nsVT & VT $\geq 30 \mathrm{~s}$ & AVB & Pause & Bradycardia \\
\hline \multirow[t]{3}{*}{ DY after 24 hours } & $\mathrm{N}$ & 127 & 354 & 87 & 194 & 2 & 107 & 111 & 224 \\
\hline & DY, \% & 17.84 & 28.15 & 40.09 & 11.39 & 11.11 & 41.63 & 20.40 & 28.72 \\
\hline & $95 \% \mathrm{Cl}$ & 15.17-20.92 & $25.72-30.76$ & $33.92-46.93$ & $9.97-13.00$ & $2.90-37.58$ & $35.88-47.91$ & $17.25-24.04$ & $25.68-32.03$ \\
\hline \multirow[t]{3}{*}{ DY after 48 hours } & $\mathrm{N}$ & 201 & 505 & 106 & 343 & 2 & 128 & 178 & 322 \\
\hline & DY, \% & 28.22 & 40.18 & 48.85 & 20.14 & 11.11 & 49.81 & 32.72 & 41.28 \\
\hline & $95 \% \mathrm{Cl}$ & $25.00-31.75$ & $37.49-42.99$ & $42.43-55.69$ & $18.31-22.13$ & $2.90-37.58$ & $43.88-56.07$ & $28.95-36.84$ & $37.92-44.82$ \\
\hline \multirow[t]{3}{*}{ DY after 5 days } & $\mathrm{N}$ & 343 & 758 & 145 & 686 & 8 & 161 & 317 & 483 \\
\hline & DY, \% & 48.10 & 60.31 & 66.82 & 40.28 & 44.44 & 62.65 & 58.27 & 61.92 \\
\hline & $95 \% \mathrm{Cl}$ & $44.43-51.91$ & $57.58-63.06$ & $60.53-73.00$ & $38.00-42.65$ & $25.25-69.49$ & $56.76-68.54$ & $54.17-62.44$ & $58.53-65.33$ \\
\hline \multirow[t]{3}{*}{ DY after 7 days } & $\mathrm{N}$ & 409 & 857 & 159 & 887 & 10 & 181 & 368 & 536 \\
\hline & DY, \% & 57.31 & 68.25 & 73.27 & 52.08 & 55.56 & 70.43 & 67.65 & 68.72 \\
\hline & $95 \% \mathrm{Cl}$ & $53.64-61.04$ & $65.63-70.84$ & $67.26-78.97$ & $49.73-54.48$ & $34.88-78.42$ & $64.78-75.89$ & $63.70-71.54$ & $65.45-71.94$ \\
\hline \multirow[t]{3}{*}{ DY after 14 days } & $\mathrm{N}$ & 590 & 1106 & 186 & 1308 & 14 & 223 & 472 & 669 \\
\hline & DY, \% & 82.75 & 88.05 & 85.71 & 76.81 & 77.78 & 86.77 & 86.76 & 85.77 \\
\hline & $95 \% \mathrm{Cl}$ & $79.82-85.48$ & $86.16-89.80$ & $80.71-89.98$ & 74.78-78.78 & $57.12-93.09$ & $82.32-90.57$ & $83.77-89.45$ & $83.22-88.11$ \\
\hline \multirow[t]{3}{*}{ DY after 30 days } & $\mathrm{N}$ & 713 & 1256 & 217 & 1703 & 18 & 257 & 544 & 780 \\
\hline & DY, \% & 100.00 & 100.00 & 100.00 & 100.00 & 100.00 & 100.00 & 100.00 & 100.00 \\
\hline & $95 \% \mathrm{Cl}$ & - & - & - & - & - & - & - & - \\
\hline
\end{tabular}

Abbreviations: ABV, atrioventricular blocks; AF, atrial fibrillation; Cl, confidence interval; DY, diagnostic yield; nsVT, non-sustained ventricular tachycardias; PVC, premature ventricular contractions; VT, ventricular tachycardias

Table 2. Diagnostic yield in men calculated after 24 hours, 48 hours, 5, 7, and 14 days

\begin{tabular}{|c|c|c|c|c|c|c|c|c|c|}
\hline \multicolumn{2}{|c|}{ Arrhythmia } & AF $\leq 1 \%$ & $A F \leq 10 \%$ & PVCs & nsVT & VT $\geq 30 \mathrm{~s}$ & AVB & Pause & Bradycardia \\
\hline \multirow[t]{3}{*}{ DY after 24 hours } & $\mathrm{N}$ & 99 & 301 & 162 & 406 & 6 & 177 & 179 & 426 \\
\hline & DY, \% & 18.66 & 28.84 & 44.51 & 17.17 & 17.14 & 49.44 & 26.68 & 35.89 \\
\hline & $95 \% \mathrm{Cl}$ & $15.54-22.33$ & $26.16-31.73$ & $39.57-49.77$ & $15.71-18.75$ & $8.09-34.23$ & $44.40-54.73$ & $23.50-30.19$ & $33.23-38.69$ \\
\hline \multirow[t]{3}{*}{ DY after 48 hours } & $\mathrm{N}$ & 150 & 423 & 198 & 638 & 13 & 213 & 247 & 571 \\
\hline & DY, \% & 28.29 & 40.55 & 54.40 & 26.98 & 37.14 & 59.50 & 36.81 & 48.10 \\
\hline & $95 \% \mathrm{Cl}$ & $24.59-32.42$ & $37.60-43.64$ & $49.37-59.58$ & $25.24-28.81$ & $23.53-55.23$ & $54.46-64.60$ & $33.29-40.58$ & $45.30-50.99$ \\
\hline \multirow[t]{3}{*}{ DY after 5 days } & $\mathrm{N}$ & 266 & 639 & 265 & 1125 & 19 & 265 & 384 & 797 \\
\hline & DY, \% & 50.10 & 61.22 & 72.80 & 47.57 & 54.29 & 74.02 & 57.23 & 67.14 \\
\hline & $95 \% \mathrm{Cl}$ & $45.84-54.52$ & $58.23-64.22$ & $68.16-77.27$ & $45.58-49.60$ & $38.95-71.10$ & 69.39- 78.45 & $53.52-60.99$ & $64.46-69.80$ \\
\hline \multirow[t]{3}{*}{ DY after 7 days } & $\mathrm{N}$ & 319 & 735 & 290 & 1366 & 23 & 285 & 446 & 892 \\
\hline & DY, \% & 60.12 & 70.37 & 79.67 & 57.76 & 65.71 & 79.61 & 66.47 & 75.15 \\
\hline & $95 \% \mathrm{Cl}$ & $55.89-64.38$ & $67.55-73.15$ & $75.39-83.63$ & $55.78-59.76$ & $50.21-80.66$ & $75.29-83.61$ & $62.89-70.02$ & $72.66-77.57$ \\
\hline \multirow[t]{3}{*}{ DY after 14 days } & $\mathrm{N}$ & 429 & 902 & 339 & 1932 & 25 & 319 & 574 & 1073 \\
\hline & DY, \% & 80.94 & 86.42 & 93.13 & 81.69 & 71.43 & 89.11 & 85.54 & 90.40 \\
\hline & $95 \% \mathrm{Cl}$ & $77.42-84.23$ & $84.23-88.44$ & $90.23-95.43$ & $80.11-83.22$ & $56.16-85.09$ & $85.62-92.07$ & $82.77-88.08$ & 88.54-91.99 \\
\hline \multirow[t]{3}{*}{ DY after 30 days } & $\mathrm{N}$ & 530 & 1044 & 364 & 2365 & 35 & 358 & 671 & 1187 \\
\hline & DY, \% & 100 & 100 & 100 & 100 & 100 & 100 & 100 & 100 \\
\hline & $95 \% \mathrm{Cl}$ & - & - & - & - & - & - & - & - \\
\hline
\end{tabular}

Abbreviations: see Table 1

cantly higher in men than in women is less documented. In light of this, we hypothesize that men are not only generally more likely than women to suffer from these distinct arrhythmias but also that in men some arrhythmic episodes are more frequent and thus diagnosed earlier.

Moreover, we found that, except AVB and sustained ventricular tachycardias, younger patients experienced arrhythmias at least as often as older patients. Further research is needed to identify reasons for sex and age differences in arrhythmia detection.

In conclusion, an extension of cardiac monitoring to 30 days leads to a substantial increase in diagnostic yield for all studied arrhythmias. In patients for whom the diagnosis of arrhythmia is likely to change clinical management, even a recording duration of 14 days is inadequate since one in every ten patients would not be diagnosed. Extended monitoring with modern ECG devices enables improved accuracy of cardiac arrhythmias detection, leading to substantial benefits both for patients and for the healthcare service.

\section{Strengths and limitations of the study}

The main strength of the presented study is the very large number of subjects included in the analysis. This enabled a reliable calculation of the diagnostic yield of both infrequent and serious arrhythmias. Furthermore, to estimate the diagnostic yield of the traditional Holter monitoring, we did not use a separate dataset collected with a Holter device. Instead, we selected shorter recordings from the 


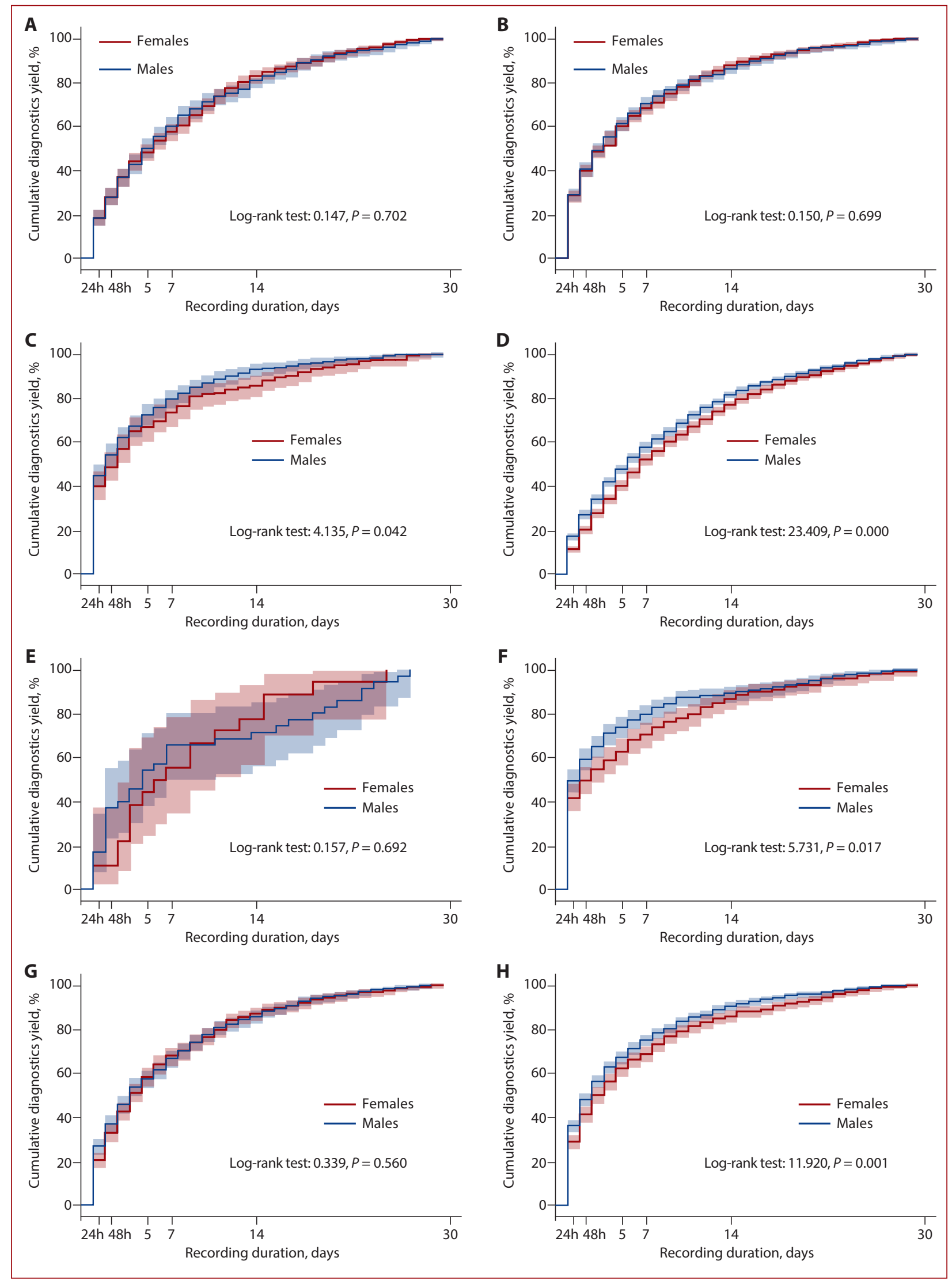

Figure 4. Cumulative DY in women (red) and men (blue) presented for $\mathbf{A}$. atrial fibrillation $\leq 1 \%$, B. atrial fibrillation $\leq 10 \%$, C. premature ventricular contractions, D. non-sustained ventricular tachycardia, E. sustained ventricular tachycardia, F. atrioventricular blocks, G. pauses, H. bradycardia 

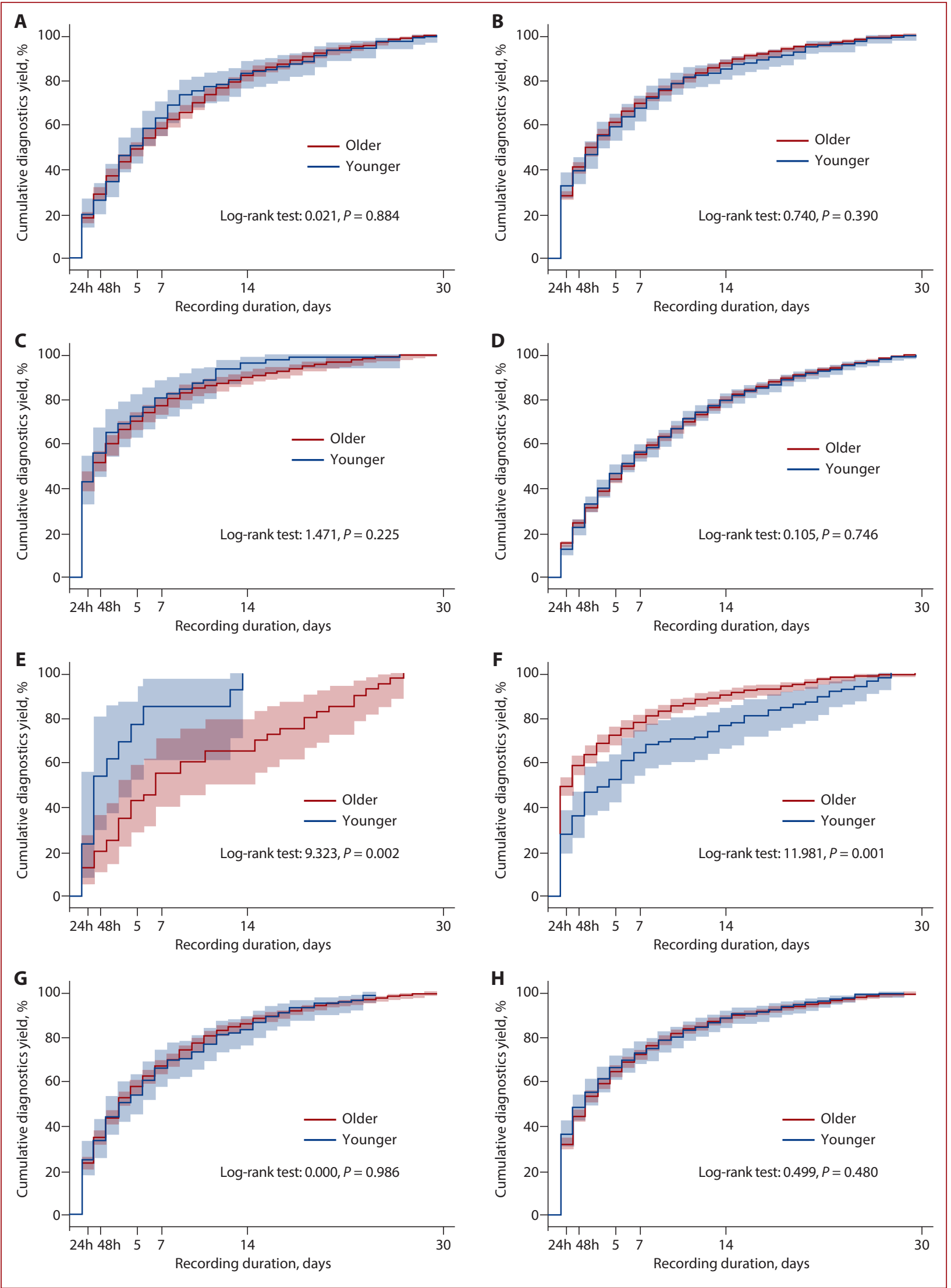

Figure 5. Cumulative diagnostic yield in older patients (red) and younger (blue) presented for A. atrial fibrillation $\leq 1 \%$, B. atrial fibrillation $\leq 10 \%$, C. premature ventricular contractions, D. non-sustained ventricular tachycardia, E. sustained ventricular tachycardia F. atrioventricular blocks, G. pauses, H. bradycardia 
long PocketECG ${ }^{\mathrm{TM}}$ sessions. As a result, we avoided introducing potential confounding factors such as different data quality, electrodes placement, or arrhythmia detection accuracy. However, some limitations of the presented study also should be noted. The main limitation of the study is the heterogeneity of the investigated sample and the lack of more detailed information regarding the study participants. It is plausible that considering factors such as a patient's medical history, admission criteria, or received treatment may bring insights into the relationship between monitoring duration and the diagnostic yield. It might also shed light on which patient populations could benefit most from extended monitoring.

\section{Article information}

Conflict of interest: MJD: Chairman of the Supervisory Board, equity interest in Medicalgorithmics SA. LSBJ: scientific collaboration. LSBJ does not receive any economical/financial reward for her activity. NEN, OW, AMG, MAS: Employee of Medicalgorithmics SA.

Open access: This article is available in open access under Creative Common Attribution-Non-Commercial-No Derivatives 4.0 International (CC BY-NC-ND 4.0) license, allowing to download articles and share them with others as long as they credit the authors and the publisher, but without permission to change them in any way or use them commercially. For commercial use, please contact the journal office at kardiologiapolska@ptkardio.pl.

\section{REFERENCES}

1. Thiruganasambandamoorthy $\mathrm{V}$, Rowe $B$, Sivilotti $M$, et al. Duration of electrocardiographic monitoring of emergency department patients with syncope. Circulation. 2019; 139(11): 1396-1406, doi: 10.1161/circulationaha.118.036088, indexed in Pubmed: 30661373.

2. Hsia B, Greige N, Patel S, et al. Determining the optimal duration for premature ventricular contraction monitoring. Heart Rhythm. 2020; 17(12):21192125, doi: 10.1016/j.hrthm.2020.07.013, indexed in Pubmed: 32679267.

3. Cheung CC, Kerr CR, Krahn AD. Comparing 14-day adhesive patch with 24-h Holter monitoring. Future Cardiol. 2014; 10(3): 319-322, doi: 10.2217/fca.14.24, indexed in Pubmed: 24976467.

4. Hayıroğlu Mi, Çinier G, Yüksel G, et al. Effect of a mobile application and smart devices on heart rate variability in diabetic patients with high cardiovascular risk: A sub-study of the LIGHT randomized clinical trial. Kardiol
Pol. 2021; 79(11): 1239-1244, doi: 10.33963/KP.a2021.0112, indexed in Pubmed: 34599495.

5. Al-Khatib SM, Stevenson WG, Ackerman MJ, et al. 2017 AHA/ACC/HRS guideline for management of patients with ventricular arrhythmias and the prevention of sudden cardiac death: Executive summary: A Report of the American College of Cardiology/American Heart Association Task Force on Clinical Practice Guidelines and the Heart Rhythm Society. Heart Rhythm. 2018; 15(10): e190-e252, doi: 10.1016/j.hrthm.2017.10.035, indexed in Pubmed: 29097320.

6. Dziubiński M. PocketECG: a new continuous and real-time ambulatory arrhythmia diagnostic method. Cardiol J. 2011; 18(4): 454-460, indexed in Pubmed: 21769831.

7. U.S. Food and Drug Administration. Premarket Notification. Available online: www.accessdata.fda.gov/scripts/cdrh/cfdocs/cfPMN/pmn. cfm?ID=K090037. [Access: November 10, 2020].

8. Farzam K, Richards JR. Premature ventricular contraction. Treasure Island, FL, USA, StatPearls Publishing 2021.

9. Koester $C$, Ibrahim $A M$, Cancel $M$, et al. The ubiquitous premature ventricular complex. Cureus. 2020; 12(1): e6585, doi: 10.7759/cureus.6585, indexed in Pubmed: 32051798.

10. Ventricular tachycardia. Treasure Island, FL, USA, StatPearls Publishing 2020.

11. Katritsis DG, Zareba W, Camm AJ. Nonsustained ventricular tachycardia. J Am Coll Cardiol. 2012;60(20): 1993-2004, doi: 10.1016/j.jacc.2011.12.063, indexed in Pubmed: 23083773.

12. Kashou AH, Goyal A, Nguyenet $T$, et al. Atrioventricular Block. Treasure Island, FL, USA, StatPearls Publishing 2017.

13. Kusumoto FM, Schoenfeld MH, Barrett C, et al. 2018 ACC/AHA/HRS guideline on the evaluation and management of patients with bradycardia and cardiac conduction delay: a report of the American College of Cardiology/American Heart Association Task Force on Clinical Practice Guidelines and the Heart Rhythm Society. Circulation. 2019; 140(8): e382-e482, doi: 10.1016/j.jacc.2018.10.043.

14. Lifelines. Available online: https://lifelines.readthedocs.io/en/latest/index. html. [Access: December 16, 2021].

15. Rich JT, Neely JG, Paniello RC, et al. A practical guide to understanding Kaplan-Meier curves. Otolaryngol Head Neck Surg. 2010; 143(3):331-336, doi: 10.1016/j.otohns.2010.05.007, indexed in Pubmed: 20723767.

16. Miettinen OS. Survival analysis: up from Kaplan-Meier-Greenwood. Eur J Epidemiol. 2008; 23(9):585-592, doi: 10.1007/s10654-008-9278-7, indexed in Pubmed: 18780149.

17. Mantel N. Evaluation of survival data and two new rank order statistics arising in its consideration. Cancer Chemother Rep. 1966; 50(3): 163-170, indexed in Pubmed: 5910392.

18. Bernal O, Moro C. Cardiac arrhythmias in women. Rev Esp Cardiol (Engl Ed). 2006; 59(6): 609-618, doi: 10.1016/s1885-5857(07)60011-5. 